\title{
¿Cuál es el perfil de los usuarios de BiciMAD? Análisis cluster ex-ante y ex-post a la inauguración del servicio
}

\author{
Andrés García-Martínez \\ Investigador, Centro de Investigación del Transporte TRANSyT. Universidad Politécnica \\ de Madrid, España. \\ Roberto Lillo \\ Estudiante de Máster. Politecnico di Torino, Italia.
}

\section{RESUMEN}

A pesar de que la mayoría los sistemas públicos de bicicletas en España comienzan a desarrollarse a partir de 2007, en la actualidad se encuentran en un momento de pleno desarrollo, pues aún siguen ampliando su área de servicio e incorporando nuevos elementos. Estos sistemas adquieren una tremenda importancia como herramientas para la promoción de la bicicleta, además de ofrecer una alternativa extra dentro del entramado de posibilidades de transporte que presenta una ciudad. En junio de 2014, Madrid implantó su propio sistema público de bicicletas (BiciMAD). Este servicio resultó innovador en muchos sentidos, pues contaba con la singularidad de ofrecer asistencia eléctrica al pedaleo. Esta tecnología mitiga uno de los principales obstáculos al usar la bicicleta: la orografía. Por otra parte, el servicio incorpora un novedoso sistema de bonificaciones, una aplicación móvil avanzada y además, es el primero en ámbito nacional que cobra por uso desde la primera media hora.

El artículo tiene el objetivo de estudiar los perfiles de usuarios de BiciMAD, mediante un análisis cluster que clasifica a los individuos según la frecuencia de uso del servicio. Para ello, se realizaron dos olas de encuestas a usuarios del sistema: una ex-ante a su inauguración (en mayo de 2014) y otra tras un año de funcionamiento (en junio de 2015). Los resultados arrojan 3 perfiles de usuarios claramente diferenciados (ciclistas habituales, ciclistas esporádicos y nuevos usuarios). El estudio aporta evidencias sobre un aumento en el uso de la bicicleta privada para todos los motivos de viaje de los abonados a BiciMAD, a la vez que escenifica un uso del sistema predominantemente para viajes de movilidad obligada -trabajo o estudios-, especialmente entre nuevos usuarios atraídos por las características del sistema y las innovaciones ofrecidas.

\section{INTRODUCCIÓN}

En los últimos años, la planificación ciclista está ganando popularidad como modo de transporte sostenible y saludable para reducir la congestión en las ciudades y las emisiones asociadas. En esta línea, los sistemas públicos de bicicletas se conciben como un modo alternativo de transporte, y como una herramienta de promoción de la bicicleta. Fishman et al. (2014) muestran que hasta el $20 \%$ los viajes en estos sistemas provienen del coche particular en ciudades como Melbourne, Brisbane o Minnesota. En otras ciudades, este 
porcentaje se sitúa por debajo del 10\% (Washington D. C. y Londres). En estas ciudades, se ha conseguido una reducción media en la distancia recorrida por coches particulares de 252.844 kilómetros. Desde el punto de vista medioambiental también resulta beneficioso, pues un ciclista libera alrededor de 21 gramos de $\mathrm{CO}_{2}$ eq por viajeros-kilómetro, mientras que un coche particular y un autobús liberan alrededor de 2.712 y 1.013 gramos de $\mathrm{CO}_{2} \mathrm{eq}$ por vehículo-kilómetro, respectivamente (Mrkajic et al., 2015). Estos resultados refuerzan la introducción de sistemas públicos de bicicletas como medida que persigue la consecución de ciudades más sostenibles.

En 2014 existían más de 600 sistemas públicos de bicicletas operativos en todo el mundo, un número que sin duda seguirá creciendo durante los próximos años (Wald, 2014). En España, los servicios públicos de bicicletas están comenzando a proliferar, encontrándose en un momento de desarrollo (Observatorio de la Movilidad Metropolitana, 2015). En el caso de Madrid, la ciudad comenzó a interesarse en 2008 por implantar su propio sistema público de bicicletas (tras la aprobación del Plan Director de Movilidad Ciclista de Madrid), siendo en 2010 cuando presentó una propuesta formal. Sin embargo, la crisis económica pospuso su inauguración hasta el 23 de junio de 2014, bajo el nombre de BiciMAD. El servicio cuenta con peculiaridades que lo distinguen del resto de sistemas públicos de bicicletas operativos en todo el mundo:

- Tecnología eléctrica en toda su flota, que proporciona al usuario asistencia al pedaleo.

- Las bicicletas pueden rastrearse con un GPS localizador.

- Redistribución inteligente: sistema de descuentos si los usuarios utilizan una bicicleta de una estación excedentaria y la devuelven en una deficitaria.

- Puntos de información en cada estación y desarrollo de una aplicación móvil, que detallan la ocupación de las bases y las bicicletas disponibles en tiempo real.

- Cobro por uso desde el primer minuto y según periodos de media hora, como medida de gestión de la movilidad, para desincentivar viajes realizados en transporte público o a pie.

\section{FACTORES QUE INFLUYEN EN LOS HÁBITOS DE MOVILIDAD CICLISTA}

Para comprender qué factores influyen en la demanda de un sistema público de bicicletas, es necesario incorporar preguntas sobre la consideración hacia el servicio, al ser el aspecto que determina en mayor grado su intención de uso. Así se desprende del estudio de Rodríguez-Priego y Porcu (2013), que afirman que los factores que más influyen son la actitud hacia la bicicleta, la sensación de control percibida y las normas subjetivas personales. En este aspecto, también hay que tener en cuenta que la familiaridad influye en la percepción de los ciudadanos, tal y como refleja Rondinella (2015). En su tesis, concluye que las personas con menos familiaridad tienen una consideración menor de los aspectos positivos de la bicicleta, y de la misma forma, se muestran más preocupados sobre sus factores negativos. No obstante, la intención de uso puede distar de los hábitos de uso reales. Kerr et al. (2010) indican que los usuarios habituados a usar varios modos de transporte 
cotidianamente tienen mayor predisposición a usar cualquier otro modo, a diferencia de los que utilizan asiduamente el vehículo privado. En el uso de la bicicleta también influyen otros factores distintos de normas subjetivas, percepciones y hábitos de uso. Winters et al., (2013) consideran en su estudio que los factores que más influencia tienen sobre la movilidad ciclista son las infraestructuras ciclistas existentes, su calidad, la accesibilidad de la red ciclista, la topografía y los usos del suelo.

Por otra parte, la convivencia de sistemas públicos de bicicletas y las bicicletas privadas tienen consecuencias en ambos sentidos. Fishman et al. (2014) muestran en sus casos de estudio que casi un $10 \%$ de los viajes realizados en estos sistemas provienen de la bicicleta privada. Y en sentido contrario, de la tesis de Castro (2011) se desprende que en países sin cultura ciclista previa, la implantación de un sistema público de bicicletas ha contribuido en algunos casos a duplicar el número de viajes en bicicleta ya existentes antes del sistema. No obstante, en la bibliografía consultada son escasos los estudios que profundicen en esta relación sistemas públicos de bicicletas-bicicletas privadas, máxime en el caso de sistemas públicos de bicicletas innovadores como BiciMAD.

Siguiendo esta línea, el artículo tiene el objetivo de analizar el impacto de la introducción de un sistema público de bicicletas en los hábitos de movilidad ciclista en la ciudad de Madrid, teniendo en cuenta la particularidad de que cuentan con tecnología eléctrica de asistencia al pedaleo. Al ser uno de los primeros sistemas con esta característica en Europa y todo el mundo, los resultados contribuyen a conocer mejor su funcionamiento y sus efectos en la movilidad. Concretamente, se intenta discernir los distintos perfiles de usuarios de los abonados a BiciMAD tras un año de su inauguración, y a su vez, contrastar si el sistema fomenta entre sus usuarios el uso de la bicicleta privada.

\section{METODOLOGÍA DEL ANÁLISIS}

\subsection{Campaña de encuestas}

Para alcanzar los objetivos de la investigación, se realizaron una encuesta ex-ante a la inauguración del servicio (mayo de 2014), y una encuesta ex-post tras un año de funcionamiento (junio de 2015). Las encuestas se dividieron en tres partes: (1) uso de la bicicleta y opinión sobre los factores que condicionan su uso, (2) opinión sobre el sistema de bicicleta pública en Madrid y su utilización (potencial, en el caso de la encuesta ex-ante) y (3) características personales (datos demográficos y socioeconómicos). En ambas encuestas se incidió sobre el uso de la bicicleta privada, y sobre el uso (hipotético en el caso de la encuesta ex-ante) del sistema BiciMAD.

En la encuesta ex-ante la población objetivo se escogió aleatoriamente entre usuarios de diferentes modos de transporte, mientras que en la encuesta ex-post se centró la atención especialmente en abonados de BiciMAD. Finalmente, se obtuvieron un total de 2.041 y 472 respuestas válidas en la primera y segunda encuesta, respectivamente. Del total de la muestra 
obtenida, el artículo se centra exclusivamente en los abonados a BiciMAD -en la encuesta ex-ante, en aquellos que declaraban intencionalidad de abonarse en un futuro inmediato-. De esta forma, la muestra final considerada se compone de 404 y 381 respuestas válidas en la primera y segunda encuesta, respectivamente.

\subsection{Análisis cluster}

El último paso de la metodología comprende el análisis estadístico de los datos. En relación con el objetivo perseguido, se eligió el análisis cluster como el método más apropiado para identificar y analizar diferentes perfiles de usuario -grupos o conglomerados- abonados al servicio BiciMAD. El análisis cluster persigue reducir la dimensión de la muestra inicial mediante el estudio de similitudes y diferencias entre cada uno de los grupos iniciales. Posteriormente debe elegirse la técnica a emplear para formar los conglomerados. Ésta puede ser jerárquica si se persigue obtener conglomerados con características lo suficientemente homogéneas -mediante un contraste de significación-, o no jerárquica si el número de grupos es decidido con anterioridad.

En primer lugar, se eligió como punto de partida la muestra obtenida en la encuesta ex-post. Las variables introducidas responden a la frecuencia de uso (diario, algunas veces por semana, una vez por semana, una vez al mes, nunca) de BiciMAD para distintos motivos de viaje (trabajo o centro de estudios, compras o pequeños recados, visitas a amigos o familiares, ocio y deporte, y salir de noche). De esta forma, los conglomerados creados responden a perfiles reales de usuario del servicio, a diferencia de los pertenecientes a la encuesta ex-ante que únicamente mostraban predisposición de uso. De las técnicas jerárquicas, se eligió el método de Ward con distancia euclídea al cuadrado, lo que significa que los miembros de los clusters se determinan calculando la suma de las desviaciones al cuadrado de los elementos de la media del cluster (Nardo et al., 2005). El método es un procedimiento de aglomeración donde un par de grupos se combinan en cada paso. A medida que el proceso avanza, las similitudes de los individuos que pertenecen al mismo conglomerado disminuyen y las distancias de enlace aumentan.

La decisión de adoptar un número de clusters óptimo es subjetiva. No obstante, para tomar esta decisión conviene analizar los gráficos de aglomeración de coeficientes y las distancias de enlace a lo largo del proceso (Milligan y Cooper, 1985). Teniendo en cuenta estas consideraciones, se decidió clasificar a los individuos en 3 conglomerados -formados, respectivamente, por 137, 121 y 123 individuos-. La Figura 1 muestra el dendograma del análisis cluster; las distancias de enlace para cada uno de los 3 conglomerados son aceptables. A partir del punto óptimo, estas distancias aumentan considerablemente, donde el método adoptado forma 2 conglomerados. 


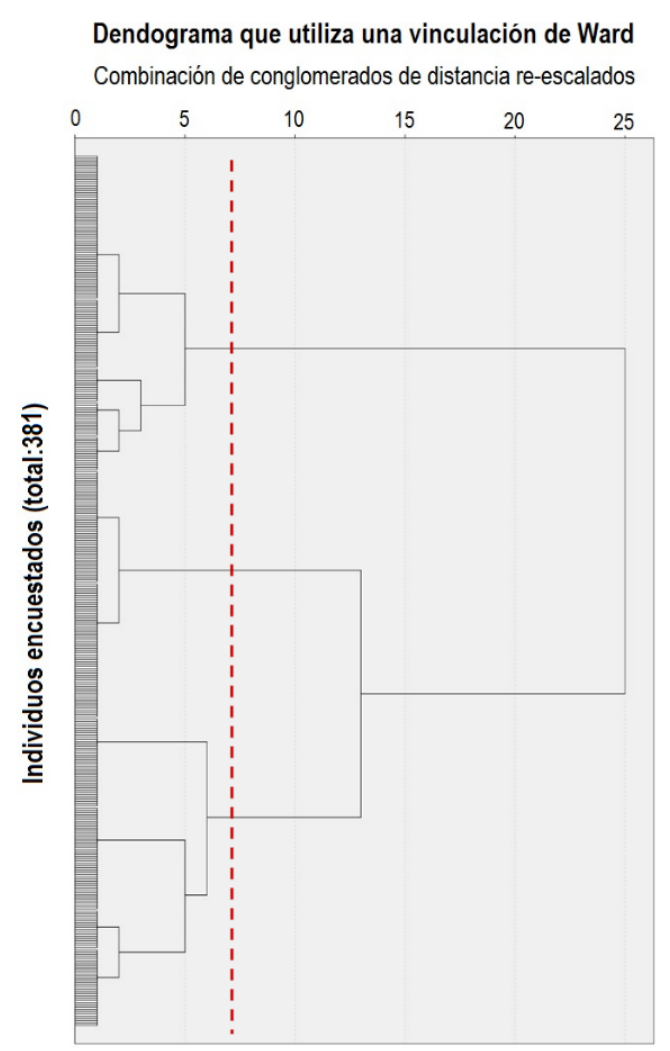

Fig. 1 - Dendograma del análisis cluster a partir de los datos de la encuesta ex-post.

Posteriormente, con el objetivo de identificar y comparar estos conglomerados con los datos de la encuesta ex-ante, se decidió aplicar en ella un análisis cluster con el método de kmedias. Este método de agrupación es no jerárquico, debiendo establecer con anterioridad el número de grupos requeridos. El algoritmo parte de la situación inicial definida moviendo los elementos entre los grupos, persiguiendo minimizar la varianza de los elementos dentro de los grupos y maximizar la varianza de los elementos fuera de los grupos (Nardo et al., 2005).

Al igual que en el análisis cluster de la encuesta ex-post, se introdujeron las frecuencias futuras de uso de BiciMAD -de los individuos que mostraron voluntad en inscribirse al servicio- según diferentes motivos de viaje, y se fijó el número de clusters deseados en 3. Los conglomerados obtenidos estaban formados por 113, 192 y 99 individuos. El análisis ANOVA de las variables muestra en todos los casos una significancia inferior a 0,05 , con valores altos del estadístico $\mathrm{F}$, lo que indica que todas las variables introducidas contribuyen a la clasificación adoptada de 3 clusters. Este hecho prueba la coherencia de la clasificación. Además, los grupos guardan similitud con los formados a partir de la encuesta ex-post, lo que indica la idoneidad del análisis cluster con el método de k-medias.

A partir de este punto, el análisis llevado a cabo permite comparar los grupos de las encuestas ex-ante y ex-post entre sí. También es posible estudiar otras características para establecer similitudes y diferencias entre conglomerados. El artículo considera la frecuencia de uso de 
la bicicleta privada un factor relevante y presenta su estudio, con el objetivo de aportar más información sobre el uso general de bicicleta en cada uno de los grupos.

\section{RESULTADOS}

Este apartado recoge los resultados obtenidos de las campañas de encuestas y los análisis estadísticos posteriores, diferenciando entre dos subapartados: (1) los resultados del análisis cluster, y (2) la influencia de la introducción del sistema sobre el uso de la bicicleta privada.

\subsection{Resultados del análisis cluster}

Este subapartado incluye en primer lugar la caracterización de los clusters formados a partir de la frecuencia de uso del servicio en la encuesta ex-post (2015). El número de individuos pertenecientes a cada cluster se muestra en la Tabla 1, además de la esperanza y su desviación típica para cada uno de los motivos de viaje. Recordemos que el análisis cluster jerárquico aplicado corresponde al método de Ward, obteniendo un total de 3 conglomerados. Los centroides -o medias- de cada cluster están comprendidos en un intervalo de 1 a 5, donde (1) representa un uso diario, (2) algunas veces por semana, (3) una vez por semana, (4) una vez al mes, y (5) nunca.

\begin{tabular}{|l|c|c|c|c|c|c|}
\hline Clusters & Muestra & $\begin{array}{c}\text { Trabajo/centro } \\
\text { de estudios }\end{array}$ & $\begin{array}{c}\text { Compras } \\
\text { o recados }\end{array}$ & $\begin{array}{c}\text { Visita a } \\
\text { amigos/familiares }\end{array}$ & $\begin{array}{c}\text { Ocio y } \\
\text { deporte }\end{array}$ & $\begin{array}{c}\text { Salir de } \\
\text { noche }\end{array}$ \\
\hline 1 & 137 & $1,6(0,4)$ & $3,0(1,0)$ & $2,6(1,0)$ & $3,0(1,3)$ & $3,4(1,5)$ \\
\hline 2 & 121 & $4,7(0,3)$ & $4,0(0,9)$ & $3,7(1,3)$ & $3,4(1,2)$ & $3,9(1,2)$ \\
\hline 3 & 123 & $1,3(0,2)$ & $4,3(0,7)$ & $4,4(0,5)$ & $4,3(0,6)$ & $4,4(0,5)$ \\
\hline
\end{tabular}

Tabla 1 - Caracterización de los clusters obtenidos de la encuesta ex-post a partir de la frecuencia de uso de BiciMAD. Análisis cluster jerárquico por el método de Ward.

El Cluster 1 -formado por 137 individuos- es el más numeroso y comprende a los usuarios del servicio que lo utilizan casi diariamente para ir a trabajar o al centro de estudios, y que realizan un uso semanal del sistema para el resto de motivos de viaje. El Cluster 2, formado por 121 individuos, se caracteriza por realizar un uso ocasional de BiciMAD para todos los motivos de viaje. El Cluster 3, en cambio, comparte similitudes con el Cluster 1, diferenciándose de éste en que sus individuos -123- utilizan BiciMAD con mayor asiduidad para trabajar o estudiar, y con una menor frecuencia para el resto de motivos de viaje.

Los resultados del análisis cluster de la encuesta ex-ante arrojan datos similares a los de la encuesta ex-post. La Tabla 2 muestra la caracterización de los clusters formados a partir de la intención de uso de BiciMAD -encuesta ex-ante 2014-, incluyendo el número de individuos de cada cluster, su esperanza y su desviación típica para cada uno de los motivos de viaje. La interpretación de los datos de la Tabla 2 es cercana a la llevada a cabo en la Tabla 1, mostrando así la similitud existente entre los clusters obtenidos a partir de las dos encuestas. El Cluster 1 -113 individuos- representa a un perfil de usuario con la intención de 
utilizar el servicio para todos los motivos de viaje. El Cluster 2 -192 individuos-, sin embargo, comprende a los usuarios que no declaran intención de usar el servicio habitualmente, salvo en determinadas ocasiones. El Cluster 3 -99 individuos- es similar al primero, salvo por el uso ocasional del servicio declarado para otros motivos de viaje.

\begin{tabular}{|l|c|c|c|c|c|c|}
\hline Clusters & Muestra & $\begin{array}{c}\text { Trabajo/centro } \\
\text { de estudios }\end{array}$ & $\begin{array}{c}\text { Compras } \\
\text { o recados }\end{array}$ & $\begin{array}{c}\text { Visita a } \\
\text { amigos/familiares }\end{array}$ & $\begin{array}{c}\text { Ocio y } \\
\text { deporte }\end{array}$ & $\begin{array}{c}\text { Salir de } \\
\text { noche }\end{array}$ \\
\hline 1 & 113 & $2,0(1,1)$ & $2,1(0,9)$ & $2,2(0,9)$ & $2,1(1,1)$ & $3,3(1,3)$ \\
\hline 2 & 192 & $4,5(0,5)$ & $3,9(0,8)$ & $3,9(0,9)$ & $3,4(1,0)$ & $4,5(0,7)$ \\
\hline 3 & 99 & $1,9(0,7)$ & $3,4(0,9)$ & $3,8(0,8)$ & $3,5(1,1)$ & $4,6(0,6)$ \\
\hline
\end{tabular}

Tabla 2 - Caracterización de los clusters obtenidos de la encuesta ex-ante a partir de la intención de uso de BiciMAD. Análisis cluster jerárquico por el método de $\mathrm{K}$ medias.

En los Clusters 1 y 3 de la encuesta ex-ante (Tabla 2), que representa la voluntad de los usuarios a utilizar BiciMAD, se aprecia cierto optimismo en el uso potencial del servicio. Los usuarios potenciales declaran una voluntad de uso del sistema que es superior a los hábitos de uso reales -encuesta ex-post (Tabla 1)-.

\subsection{Influencia de BiciMAD en el uso de la bicicleta privada}

Una vez obtenidos los clusters de la primera y segunda encuesta, resulta de interés el uso que hacen estos grupos de usuarios de la bicicleta particular. En la Tabla 3 se muestra la caracterización de estos clusters según la frecuencia de uso de la bicicleta privada, además de la esperanza y su desviación típica, en una escala comprendida entre 1 y 5 , donde (1) representa un uso diario, (2) algunas veces por semana, (3) una vez por semana, (4) una vez al mes, y (5) nunca.

\begin{tabular}{|l|c|c|c|c|c|}
\hline \multicolumn{1}{|c|}{ Clusters } & $\begin{array}{c}\text { Trabajo/centro } \\
\text { de estudios }\end{array}$ & $\begin{array}{c}\text { Compras } \\
\text { o recados }\end{array}$ & $\begin{array}{c}\text { Visita a } \\
\text { amigos/familiares }\end{array}$ & $\begin{array}{c}\text { Ocio y } \\
\text { deporte }\end{array}$ & $\begin{array}{c}\text { Salir de } \\
\text { noche }\end{array}$ \\
\hline Encuesta ex-ante & & & & & \\
\hline 1 & $3,5(1,5)$ & $3,4(1,3)$ & $3,5(1,2)$ & $2,7(1,1)$ & $4,4(1,1)$ \\
\hline 2 & $4,5(1,1)$ & $4,4(0,9)$ & $4,5(0,7)$ & $3,5(0,8)$ & $4,8(0,7)$ \\
\hline 3 & $4,0(1,5)$ & $4,1(1,1)$ & $4,3(0,9)$ & $3,2(0,9)$ & $4,8(0,6)$ \\
\hline Encuesta ex-post & & & & & \\
\hline 1 & $2,5(1,3)$ & $3,7(1,4)$ & $3,1(1,1)$ & $3,0(1,0)$ & $3,2(1,1)$ \\
\hline 2 & $3,4(1,0)$ & $4,3(0,8)$ & $3,7(0,7)$ & $3,3(0,9)$ & $3,9(0,4)$ \\
\hline 3 & $2,9(1,3)$ & $4,6(0,6)$ & $3,9(0,5)$ & $3,5(0,8)$ & $4,0(0,2)$ \\
\hline
\end{tabular}

Tabla 3 - Caracterización de los clusters obtenidos según la frecuencia de uso de la bicicleta particular.

En la encuesta ex-ante, los individuos del Cluster 1 son los únicos que utilizan la bicicleta particular, aunque con una frecuencia ocasional. Se observa cómo esta situación cambia en la encuesta ex-post tras un año de funcionamiento del sistema. La bicicleta particular pasa a 
tener más protagonismo en los hábitos de movilidad para la mayoría de motivos de viaje, alcanzando frecuencias de uso casi diarias -excepto para ir de compras y salir de noche-.

Estos resultados escenifican un aumento en el uso de la bicicleta particular, tal y como sostiene Castro (2011). En la Figura 2 se muestra el centro de cada uno de los cluster de la encuesta ex-post para cada motivo de viaje, diferenciando entre uso de BiciMAD y uso de bicicleta privada. Estos datos aportan nueva información sobre los perfiles de usuarios abonados al servicio, poniendo de relieve la coexistencia del uso de BiciMAD y de la bicicleta particular entre los tres clusters.

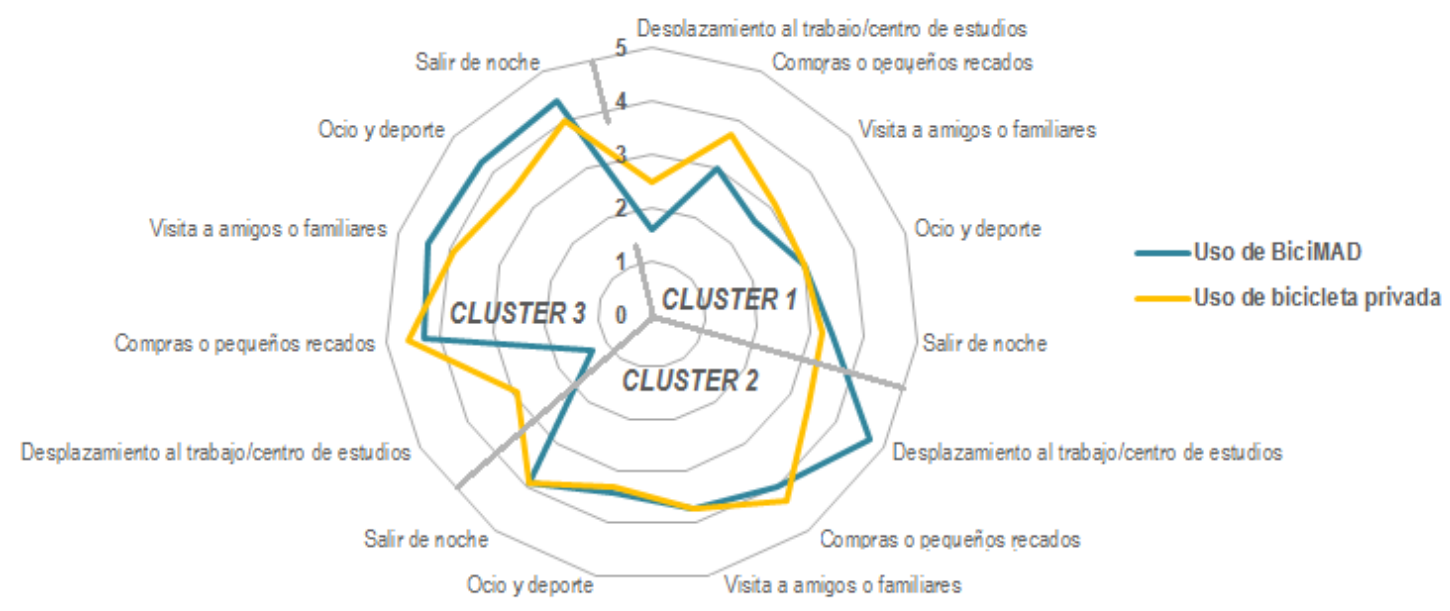

Fig. 2 - Caracterización de los clusters de la encuesta ex-post según la frecuencia de uso de BiciMAD y de la bicicleta privada.

El Cluster 1 se caracteriza por un uso más habitual de BiciMAD para todos los motivos de viaje, complementado con el uso de la bicicleta particular de forma secundaria. En el Cluster 2 , en cambio, no se aprecian diferencias significativas, salvo en los viajes al trabajo o al centro de estudios, donde el uso de la bicicleta particular es más frecuente que el de BiciMAD. Los usuarios pertenecientes al Cluster 3 generalmente prefieren utilizar su bicicleta privada con carácter semanal, salvo para los viajes de movilidad obligada donde predomina el uso de BiciMAD. El Cluster 3 es el conglomerado más interesante desde el punto de vista de los efectos de la introducción del sistema de bicicleta público, pues incorpora usuarios que en la encuesta ex-ante declaran voluntad de inscribirse al servicio pero no utilizan la bicicleta particular (Tabla 3). Tras la inauguración de BiciMAD, en cambio, lo utilizan prácticamente a diario para ir a trabajar, atraídos por las ventajas de un sistema de bicicleta público y las características innovadoras del mismo. Sin embargo, no utilizan el servicio ni su bicicleta particular para los demás motivos de viaje, quizás influenciados por la inseguridad, por el desconocimiento de la ruta, etc. En el caso de Madrid, este grupo aflora por las ventajas tecnológicas que ofrece la flota de bicicletas de BiciMAD, y supone una diferencia en cuanto a perfiles de usuarios existentes en otras ciudades con sistemas públicos de bicicletas convencionales. 
Con la información de este apartado, podemos renombrar los clusters obtenidos:

- Cluster 1: Ciclistas habituales (uso predominante de BiciMAD para todos los motivos de viaje, complementado por un uso frecuente de la bicicleta particular).

- Cluster 2: Ciclistas esporádicos (uso ocasional de la bicicleta particular para viajes de movilidad obligada y uso esporádico de BiciMAD para el resto de motivos de viaje).

- Cluster 3: Nuevos ciclistas atraídos por las ventajas de BiciMAD (uso exclusivo de BiciMAD para viajes de movilidad obligada, acompañado de un uso ocasional de la bicicleta particular para el resto de motivos de viaje).

\section{CONCLUSIONES}

El estudio aporta evidencias sobre un aumento en el uso de la bicicleta privada para todos los motivos de viaje de los abonados BiciMAD en la ciudad de Madrid, tras un año de funcionamiento del sistema. El servicio, que cuenta con particularidades que lo diferencian del resto de sistemas -tecnología eléctrica, sistema de bonificaciones y cobro por uso desde el primer minuto-, se posiciona como una alternativa de transporte en los viajes de movilidad obligada. El resto de motivos de viaje sufren un uso menor que el declarado antes de la inauguración del servicio, hecho motivado por el sistema tarifario aplicado. No obstante, el sistema también encuentra oportunidades -superiores a las declaradas- en los viajes nocturnos, al existir en la ciudad escasez de oferta de transporte público.

La investigación se centra en conocer los perfiles de usuarios abonados a BiciMAD, para lo que se propone un análisis cluster jerárquico basado en el método de Ward, que da como resultado 3 conglomerados. Del análisis se desprende que los usuarios que hacen un uso habitual u ocasional de BiciMAD para viajes de movilidad obligada anteponen su uso ante el de la bicicleta particular. Este fenómeno se explica por la innovadora introducción de tecnología eléctrica de asistencia al pedaleo y el funcionamiento del sistema, que minimizan los problemas detectados en el centro de Madrid: seguridad en el aparcamiento, aptitud física, circular junto al tráfico rodado y la orografía (Muñoz et al., 2013).

Dos de los clusters obtenidos arrojan perfiles de usuario esperados: ciclistas habituales Cluster 1- y ciclistas esporádicos -Cluster 2-. La inauguración de BiciMAD ha tenido en el primer grupo un efecto catalizador en cuanto al uso de la bicicleta particular, aumentando su frecuencia de uso en los viajes de movilidad obligada y en los viajes nocturnos. En el segundo conglomerado, sin embargo, los efectos producidos en este aspecto han resultado ser inapreciables.

Del análisis cluster también se extrae un conglomerado formado por usuarios atraídos por las ventajas tecnológicas del sistema-Cluster 3-. Estos individuos declararon la intención de inscribirse al servicio antes de su inauguración, manifestando un uso esporádico general de la bicicleta particular. Tras un año de funcionamiento de BiciMAD, lo utilizan diariamente 
y exclusivamente para viajes de movilidad obligada, sin apenas utilizar su bicicleta particular para el resto de motivos de viaje. Así, este grupo de usuarios se convierte en un objetivo potencial de futuras investigaciones, de forma que puedan conocerse las características que han atraído su atención para utilizar el servicio y las causas por las que no utilizan con más frecuencia la bicicleta privada.

Igualmente, el análisis en su conjunto supone un punto de inicio para investigaciones futuras, una vez conocidos los perfiles de usuarios abonados a BiciMAD. Como futuras líneas de investigación, se propone profundizar en las diferencias de valoración entre conglomerados relativas a infraestructura, características del servicio, sistema tarifario, etc., así como analizar sus perfiles socioeconómicos, con el objetivo de deducir las motivaciones y desavenencias que influyen sobre sus comportamientos. El estudio, no obstante, se contrastará con la información procedente de la tercera campaña de encuestas, proyectada en mayo de 2016.

\section{AGRADECIMIENTOS}

Este trabajo ha sido financiado en parte por el Ministerio de Economía y Competitividad, gracias a la concesión de una ayuda para Formación de Personal Investigador (FPI) al autor principal [BES-2014-069223]. Esta ayuda está asociada al proyecto Transfer [TRA201345239-R].

\section{REFERENCIAS}

CASTRO, A. (2011). The contribution of bike-sharing to sustainable mobility in Europe. Doctoral thesis. Vienna University of Technology.

FISHMAN, E., WASHINGTON, S. y HAWORTH, N. (2014). Bike share's impact on car use: Evidence from the United States, Great Britain, and Australia. Transportation Research Part D: Transport and Environment 31, pp. 13-20.

KERR, A., LENNON, A. y WATSON, B. (2010). The call of the road: factors predicting students' car travelling intentions and behavior. Transportation 37(1), pp. 1-13.

MILLIGAN, G. W. y COOPER, M. C. (1985). An examination of procedures for determining the number of clusters in data ser. Psychometrika 50, pp. 159-179.

MUÑOZ, B., MONZON, A. y LOIS, D. (2013). Cycling Habits and Other Psychological Variables Affecting Commuting by Bicycle in Madrid, Spain. Journal of the Transportation Research Board 2382, pp. 1-9.

MRKAJIC, V., VUKELIC, D. y MIHAJLOV, A. (2015). Reduction of CO2 emission and non-environmental co-benefits of bicycle infrastructure provision: the case of the University of Novi Sad, Serbia. Renewable and Sustainable Energy Reviews 49, pp. 232-242. 
NARDO, M., SAISANA, M., SALTELli, A., TARANTOLA, S., HOFFMAN, A. y GIOVANNINI, E. (2005). Handbook on Constructing Composite Indicators Methodology and User Guide. OECD Statistics Working Paper, Paris.

OBSERVATORIO DE LA MOVILIDAD METROPOLITANA (OMM), (2015). Informe OMM-2013. Disponible en: http://goo.gl/G9yG2J. Consultado el: 15 de enero de 2016.

RODRIGUEZ-PRIEGO, N. y PORCU, L. (2013). 'I want to hire my bicycle, I want to hire my bike': analysing the psychological drivers of students' intention to use bike hire schemes as a sustainable transportation. 7th International Technology, Education and Development Conference (INTED), pp. 5376-5382.

RONDINELLA, G. (2015). Considering Cycling for Commuting: the Role of Mode Familiarity. Doctoral thesis. Universidad Politécnica de Madrid.

WALD, C. (2014). Wheels when you need them. Science 345(6199), pp. 862-863.

WINTERS, M., BRAUER, M., SETTON, E. M. y TESCHKE, K. (2013). Mapping bikeability: a spatial tool to support sustainable travel. Environment and Planning B Planning and Design 40(5), pp. 865-883. 